\title{
First report of Rabbit hemorrhagic disease virus 2(GI.2) in China
}

\author{
Ruibin $\mathrm{Qi}^{1}$, Chunchun Meng ${ }^{1}$, Hang $\mathrm{Li}^{1}$, Jie Zhu ${ }^{1}$, Qiuhong Miao ${ }^{1}$, Jingyu Tang ${ }^{1}$, Aoxing \\ Tang ${ }^{1}$, Hongyuan $\mathrm{Guo}^{1}$, Chuncao $\mathrm{Liu}^{1}$, Chuanfeng $\mathrm{Li}^{1}$, Zongyan $\mathrm{Chen}^{1}$, Qinwen Zhang ${ }^{2}$, \\ and Guangqing $\mathrm{Liu}^{1}$
}

\author{
${ }^{1}$ Shanghai Veterinary Research Institute Chinese Academy of Agricultural Sciences \\ ${ }^{2}$ Qinghai University
}

August 3, 2020

\begin{abstract}
Rabbit haemorrhagic virus (RHDV) is a highly infectious and fatal pathogen to rabbits. Classic RHDV is mainly pathogenic to adult rabbits; however, it is not lethal to young rabbits. Since 2010, a RHDV variant has become prevalent in Europe and the Australian continent. This variant, which was named RHDV2 (GI.2/RHDVb), is highly infectious and fatal to both adult and young rabbits; moreover, its host range is broader than that of classic RHDV (GI.1/G1-6). In May 2020, the first RHDV2 case in China was reported. Here, we report and describe the first RHDV2 case in China. We amplified its complete genome sequence (named SC20-01 strain). The phylogenetic tree showed that the SC20-01 strain is mostly related to two strains isolated in Europe, which indicated that it might have spread from Europe to China. Moreover, Recombination Detection Program software showed that the SC20-01 strain is a G6/RHDV2 recombinant strain. Animal experiments showed that the SC20-01 strain is a highly fatal pathogen to specific-pathogen-free (SPF) rabbits and induces typical clinical symptoms of RHD. Our findings highlight RHDV2 emergence in China and its potential to spread widely. There is a need for more attention on the RHDV2 threat in China and the development of new diagnostic methods and vaccines for preventing the spread of RHDV2.
\end{abstract}

\section{Introduction}

Rabbit haemorrhagic disease (RHD) is a fatal infectious disease that primarily affects adult rabbits and causes great economic losses in the rabbit industry (Mitro and Krauss, 1993). The causative pathogen for RHD is RHD virus (RHDV), which belongs to the genus Lagovirus and family Calicivirus (Moussa et al., 1992). In 1984, it was first reported in China within a group of commercially-bred Angora rabbits imported from Germany. However, some studies suggested that it had spread across Europe for some time; therefore, it is difficult to clarify whether those rabbits were infected with RHDV before importation from Germany (Kerr et al., 2009). All RHDV strains are classified into one serotype; however, several different subtypes have been identified, including classic RHDV (GI.1/G1-6) and RHDV2 (GI.2/RHDVb). RHDV2 is a new variant of classic RHDV, which was first reported in north-western France in 2010 (Le Gall-Reculé et al., 2011). It has now spread across many countries in Europe, Australia, and parts of Africa (Abrantes et al., 2013; Kerr et al., 2009; Rouco et al., 2019). More recently, RHDV2 outbreaks have been reported in the United States and Canada. Compared with RHDV, RHDV2 is hosted in a wider range of rabbits and can not only infect domestic rabbits and European hares, but also a wider range of hare species, including the Sardinian cape hare (L. capensis) (Puggioni et al., 2013), Corsican hare (L. corsicanus)(Camarda et al., 2014), and European hare (L. europaeus) (Neimanis et al., 2018b). Notably, rabbits immunized with the classic RHDV vaccine can become infected with RHDV2 and die, which indicates a significant antigenic difference between RHDV2 and classic RHDV. The mechanism of its mutation remains unclear. Another unique characteristic of RHDV2 is that it can infect young rabbits and cause death with a high mortality rate (70\%-100\%). Although China is the largest rabbit breeder in the world and was the first to report RHDV, it has had no previous RHDV2 
outbreak. However, on May 2020, there was a sudden RHDV2 outbreak in Sichuan Province, China, which killed thousands of rabbits. Here, we report and describe the first RHDV2 case in China.

A large rabbit population died in two rabbit farms in Sichuan province, China (Fig 1A). Most of the dead rabbits were young rabbits (age: 30 days); however, others were adult female rabbits. Moreover, all the dead rabbits showed typical clinical signs of RHD; including anorexia, cachexia, opisthotonos, and bloody nasal discharge (Fig. 1B). The dead rabbits were autopsied to detect pathological tissue changes. The major autopsy findings were haemorrhages and congestions in the lungs, heart, and kidneys, as well as acute hepatitis and splenomegaly (Fig. 1C). For subsequent analysis, tissues, including the heart, liver, spleen, lung, kidney, and serum, were collected and stored at $-80{ }^{\circ} \mathrm{C}$.

To identify causative pathogens, the livers of the dead rabbits were homogenized with phosphate-buffered saline (PBH; pH 7.4), subjected to three freeze-thaw cycles, and centrifuged for supernatant collection. A haemagglutination assay (HA) test was performed using these supernatants and $1 \%$ human erythrocytes of blood group O. The supernatants were found to significantly agglutinate human type O erythrocytes and the HA titre was 1:800. Subsequently, the supernatants were clarified with $30 \%$ sucrose solution and washed with PBS to remove the sucrose. The collected supernatants were analysed using transmission electron microscopy (TEM), which revealed icosahedral symmetry viral particles with a 30-40 nm diameter that were consistent with those of RHDV (Fig. 2). The obtained RHDV isolation strain was named as SC20-01.

To explore the pathogen source, liver total RNA from the dead rabbits was extracted using Trizol Reagent (Invitrogen, USA) and reverse transcribed using the M-MLV reverse transcriptase (Promega, USA) The complete sequence of SC20-01 was amplified and submitted to GenBank (accession no. MT737965). From GenBank, 21 and 19 genome sequences of RHDV2 and classic RHDV, respectively, were selected. Three genome sequences of Rabbit Calicivirus (RCV) were chosen as the out-groups. A phylogenetic tree was constructed using the maximum likelihood statistical methods based on VP60 protein using MEGA5.0 software (www.megasoftware.net). The SC20-01 strain was found to belong to the RHDV2 subtype; moreover, its VP60 protein shared $97.2 \% \sim 99.5 \%$ and $86.8 \% \sim 87.8 \%$ amino acid identity with other RHDV2 subtypes and classic RHDV strains, respectively (Fig. 3A). Based on the phylogenetic tree, the SC20-01 strain was most closely related with RHDV2-NL2016 strain (accession no. MN061492) and Bremerhaven-17 strain (accession no. MN901451), which were all isolated from rabbits in Europe.

There are many genetic recombination events among RHDV; further, the inter-subtype genetic recombination events are mainly located in the ORF1s (Qi et al., 2019). We examined recombination events in the SC2001 strain using the RDB, GEGECONV, Bootscan, Maximum Chi-Square, Chimaera, Sister Scanning, and 3seq methods of the Recombination Detection Program (RDP) v4.56 software after selecting 324 genome sequences of RHDV and 3 genome sequences of RCV, except for SC20-01, from GenBank. The recombination area of the SC20-01 strain was found to be from nucleotide 5248 to 5387; moreover, its related strains were P19 (accession no. KY765610, G6) and CBAnd1 (accession no. KP090976, RHDV2) from Portugal and Spain (Table 1). Furthermore, a recombination event of the SC20-01 strain was confirmed using SimPlot with the observation of an apparent breakpoint that separated the SC20-01 genome into two regions, which was consistent with the RDP results (Fig. 3B). Two phylogenetic trees were constructed using maximum likelihood statistical methods based on two different nucleotide recombination regions (non-structural and structural protein-coding regions: 10 $5304 \mathrm{bp}$ and 5304 $7378 \mathrm{bp}$, respectively) of the SC20-01 strain using MEGA5.0 software (www.megasoftware.net). The non-structural protein-coding region of SC20-01 was more closely related with that of classic RHDV than that of RHDV2. This demonstrated that the SC20-01 strain was a classic RHDV/RHDV2 recombination strain (Fig. 3C-D).

To confirm infection with the SC20-01 strain, six 6-week-old SPF rabbits were randomly divided into two groups $(\mathrm{n}=3)$ and injected with $1 \mathrm{ml}$ of the supernatants of homogenized livers or PBS followed by observation for three days. All animal experimental procedures were approved and performed in compliance with the guidelines of the Animal Research Ethics Board of Shanghai Veterinary Institute (Shanghai, China), CAAS (no. SHVRI-SZ-20200709-01). After 24 post-infection hours, two rabbits in the experimental group showed typical clinical symptoms of RHD with one dying (Fig. 4A). The two surviving rabbits in the 
experimental group were euthanized using an intravenous injection of sodium pentobarbital to examine the tissue distribution of pathogens in various post-infection stages. After observation for 7 days, all rabbits in the control group survived and did not show any clinical RHD symptoms. All the surviving rabbits were euthanized with an intravenous injection of sodium pentobarbital. Subsequently, they were autopsied to examine tissue pathological changes and tissues were collected and stored in $4 \%$ paraformaldehyde or -80 ${ }^{\circ} \mathrm{C}$ for subsequent analysis. The tissues of rabbits infected with SC20-01 strain showed RHDV pathological changes (Fig. 4A).

To examine the viral distribution, tissue and body-fluid total RNA of rabbits infected with SC20-01 strain were extracted with Trizol Reagent (Invitrogen, USA) and reverse transcribed using the M-MLV reverse transcriptase (Promega, USA). Real-time quantitative polymerase chain reaction was used to detect viral loads in the tissues and body fluids of rabbits infected with SC20-01. Viral RNA was detected in all tissues and body fluids, including urine, the gonads, and the brain. Among those tissues and body fluids, there was a higher viral load in the liver, cholecysts, spleen, and lung, which was consistent with previous findings (Liu et al., 2015; Neimanis et al., 2018a). Furthermore, there was a high viral load in the serums of the infected rabbits (Table 2). The viral antigen was detected in both groups using immunohistochemical (IHC) analysis. The primary antibodies were mouse monoclonal antibodies targeting VP60 of RHDV2 (prepared and preserved by our laboratory) while the secondary antibody was horseradish peroxidase-conjugated goat anti-mouse antibody (Jackson ImmunoResearch, USA). Viral antigen was only detected in the tissues of rabbits infected with SC20-01 and was mainly located in the cellular cytoplasm (Fig. 4B).

In summary, we reported and identified the first RHD case caused by RHDV2, a new RHDV variant, in China through RT-PCR, HA, and IHC. Subsequently, we amplified the complete genome sequence of the SC20-01 strain and conducted a phylogenetic tree based on the amino acid sequence of VP60. We found that the strains most related to the SC20-01 strain were isolated in Europe and speculated that SC20-01 might have spread from Europe to China. Recombination detection indicated that the SC20-01 strain is an intersubtype recombination strain composed of two different RHDV subtypes. This indicated that there were genetic recombination events between classic RHDV and RHDV2. Six SPF rabbits were used to monitor the pathogenicity of the SC20-01 strain, which was found to cause death with typical clinical symptoms and RHD tissue lesions after 24 post-infection hours. Viral RNA could be detected in all tissues and body fluids of infected rabbits, including urine, which suggested viral pollution in the environments by urine and faeces. Therefore, there is a need to disinfect the environments of rabbits farms upon identification of RHD cases. Moreover, the high viral load in the brains and gonads suggested that RHDV2 could cross the bloodbrain barrier or blood testes barrier. This indicated that RHDV2 might could be vertically transmitted from female rabbits to young rabbits. There is a need to monitor the RHDV2 epidemiology in China since RHDV2 could cause death among rabbits vaccinated against classic RHDV. Moreover, it is necessary to develop new diagnostic methods and vaccines for preventing the spread of RHDV2.

\section{Acknowledgments}

This study was supported by the Key Research Project of National Science and Technology (2016YFD0500108, 2016YFD0501003), the Chinese Natural Sciences Foundation (31672572), the Fundamental Research Funds for the Central Institutes Program (2019JB06), the Science and Technology Promoting Agriculture Innovation Project of Shanghai (2019 no. 3-3), and the Foundation of Shanghai Key Laboratory of Veterinary Biotechnology (klab201712).

\section{Ethics approval and consent to participate}

Specific-pathogen-free New Zealand white rabbits were treated humanely and with regard to the alleviation of their suffering. The use of all rabbits in the immunization trials was approved by and in compliance with the guidelines of the Animal Research Ethics Board of Shanghai Veterinary Institute (CAAS).

\section{Data availability statement}

All data, models, and code generated or used during the study appear in the submitted article. 


\section{Conflicts of interest:}

None.

\section{FIGURE LEGENDS}

Table 1Recombinant outcome detected using the Recombination Detection Program v4.56.

Table 2Detection of viral loads in tissues and body fluids of rabbits infected with RHDV2 (SC20-01 strain).

Fig. 1Details of the first case of RHD caused by RHDV2 in China.(A) The location of the first case of RHD caused by RHDV2 in China. (B) Clinical signs of infected rabbits. Dead rabbits show typical clinical signs of RHD, including anorexia, cachexia, opisthotonos, and bloody nasal discharge. (C) Pathological tissue changes in infected rabbits. The major pathological changes of tissues are haemorrhages and congestions in the lungs, as well as acute hepatitis.

Fig. 2TEM images of viral particles extracted from supernatants of homogenized livers of the infected rabbits. Supernatants of homogenized livers were clarified with $30 \%$ sucrose solution. Icosahedral symmetry viral particles were observed with a diameter ranging from 30 to $40 \mathrm{~nm}$, which was consistent with those of RHDV.

Fig. 3Phylogenetic analysis of RHDV2 China isolation strain SC20-01. (A) Maximum likelihood tree for RHDV based on the VP60 protein using MEGA5.0 software. RHDV isolation strain SC20-01 belonged to the RHDV2 subtype and was most closely related with several isolation strains in Europe. (B) Nucleotide sequence divergence scans and Bootscanning of RHDV. Recombination events of the SC20-01 strain were examined using SimPlot with one apparent breakpoint that separated the SC20-01 genome into two regions being detected.

Maximum likelihood tree for RHDV based on the non-structural (10 $5304 \mathrm{bp})(\mathrm{C})$ and structural proteincoding regions (5304 $7378 \mathrm{bp})$ (D) using MEGA5.0 software. The non-structural protein-coding region of SC20-01 was more closely related with that of classic RHDV than RHDV2. Further, the results indicated that SC20-01 was a classic RHDV/RHDV2 recombination strain.

Fig. 4Animal regress experiment of SPF rabbits infected with RHDV2 China isolation strain SC20-01. (A) SPF rabbits infected with SC20-01 strain showed typical clinical signs and pathological changes of RHD. SC20-01 strain was highly fatal and could cause the death of the SPF rabbits after 24 post-injection hours. (B) IHC analysis of tissues from rabbits in both groups. Images of IHC analysis showed that VP60 of RHDV2 was present in the tissues of the rabbits in the experiment group and was mainly located in the cellular cytoplasm.

\section{Hosted file}

Table1.docx available at https://authorea.com/users/348056/articles/473531-first-report-ofrabbit-hemorrhagic-disease-virus-2-gi-2-in-china

\section{Hosted file}

Table2.docx available at https://authorea.com/users/348056/articles/473531-first-report-ofrabbit-hemorrhagic-disease-virus-2-gi-2-in-china 

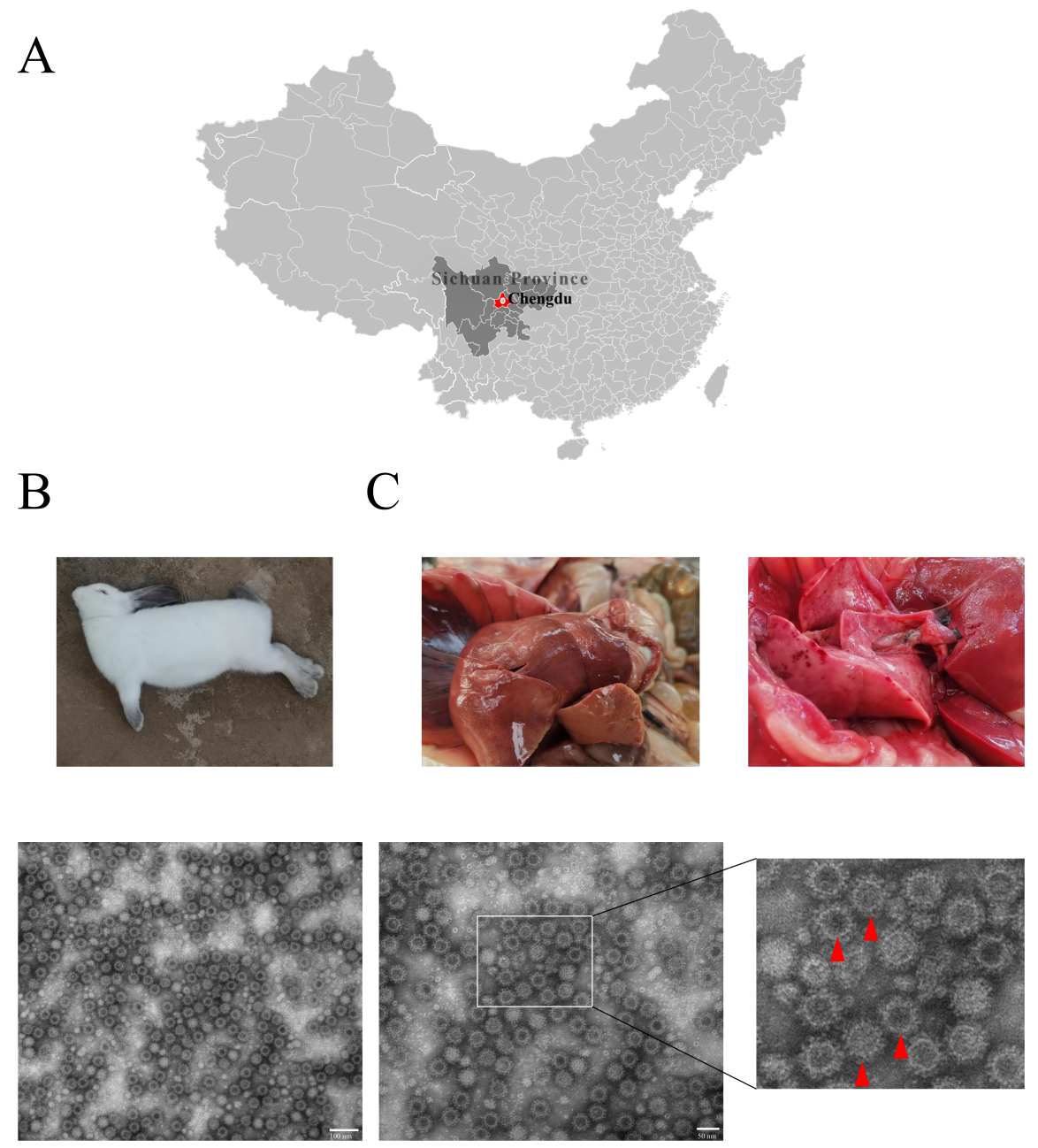
A

A

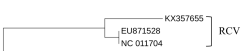

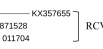

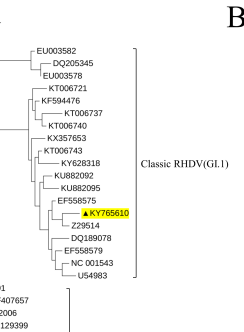

B
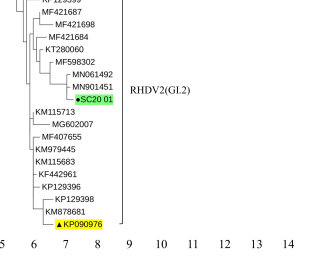

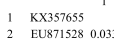

3 DQ205345 0.1430 .151

$\mathrm{KY} 7656100.1530 .1570 .048$
$\mathrm{KT} 0067370.1490 .1510 .048$
7

$K Y 6283180.1490 .1490 .0570 .030$
KU832095 0.023

KU882095 0.1470 .1510 .0530 .0300 .0260 .019

NC 0015430.1490 .1490 .0480 .0250 .0250 .0260 .0250 .014

MG602006 0.1470 .1310 .1150 .1190 .1210 .1170 .1150 .1130 .117

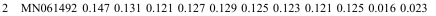

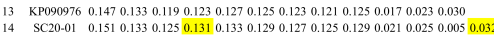

C

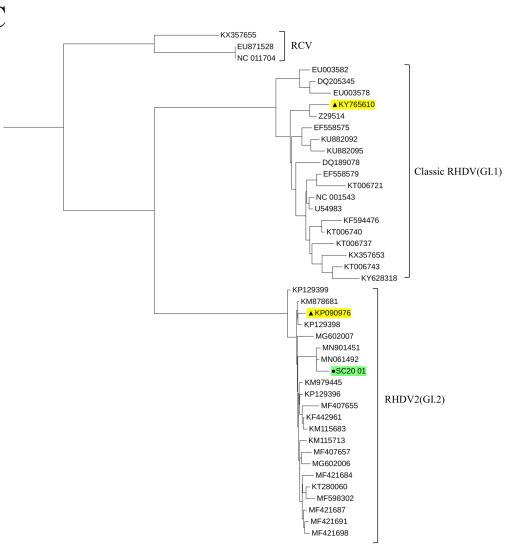

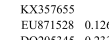

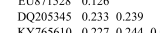

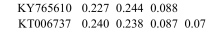

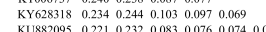

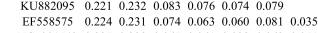

NC $0015430.2270 .2380 .0670 .0610 .038 \quad 0.0620 .0510 .039$

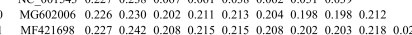

12 MN061492 0.2290 .2370 .2050 .2140 .2140 .2070 .1990 .1980 .2140 .0360 .033

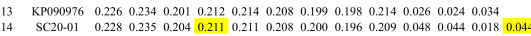

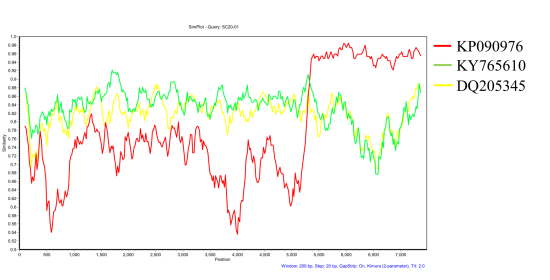

D
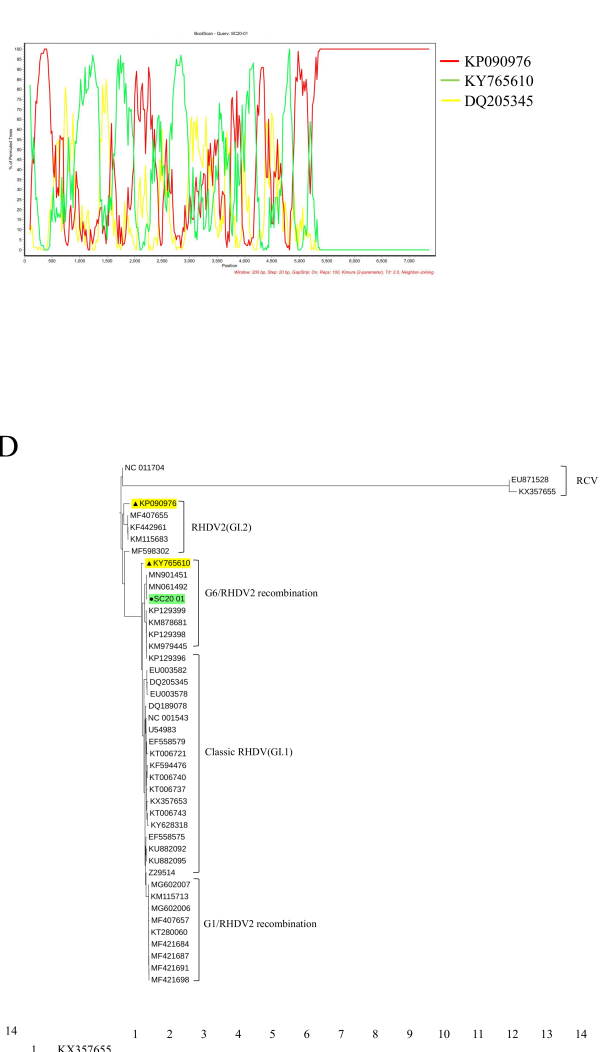

(1)

$\begin{array}{llll}1 & \text { KX357655 } & \\ 2 & \text { EU871528 } & 0.153\end{array}$

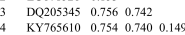

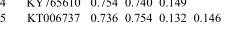

$6 \quad$ KY628318 0.740 .7530 .1480 .1550 .071

FF558575 075207401280.13500020 .1050 .032

9 NC_001543 0.7370 .7470 .1250 .1350 .0330 .0680 .0680 .063

MF421698 0.7530 .7480 .14701660 .1240 .1340 .1050 .01010 .10800034

12 MN061492 0.7580 .7350 .1720 .1460 .1570 .1630 .1560 .1510 .1480 .1660 .168

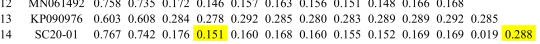



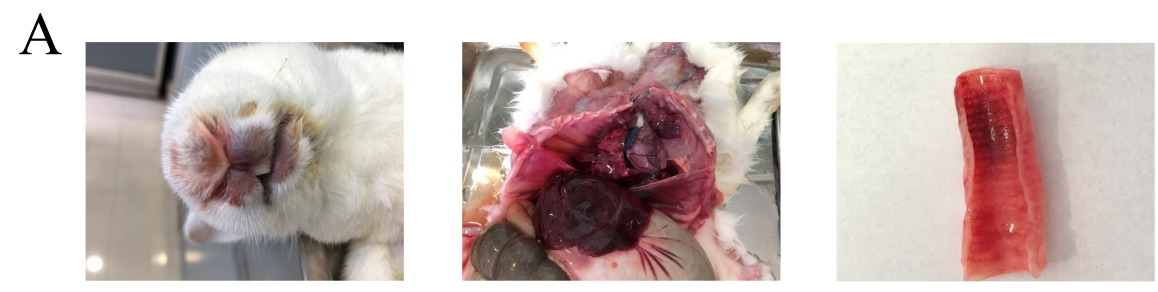

B

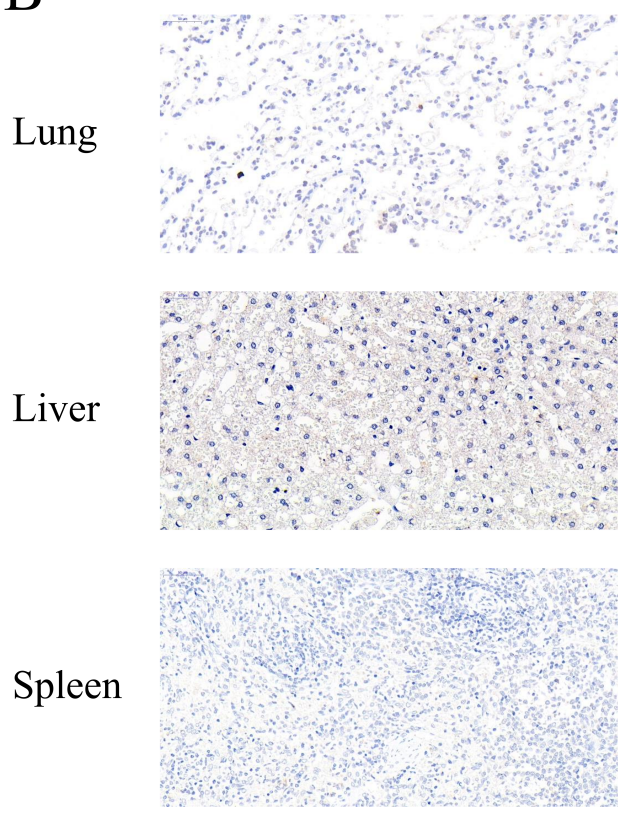

Control
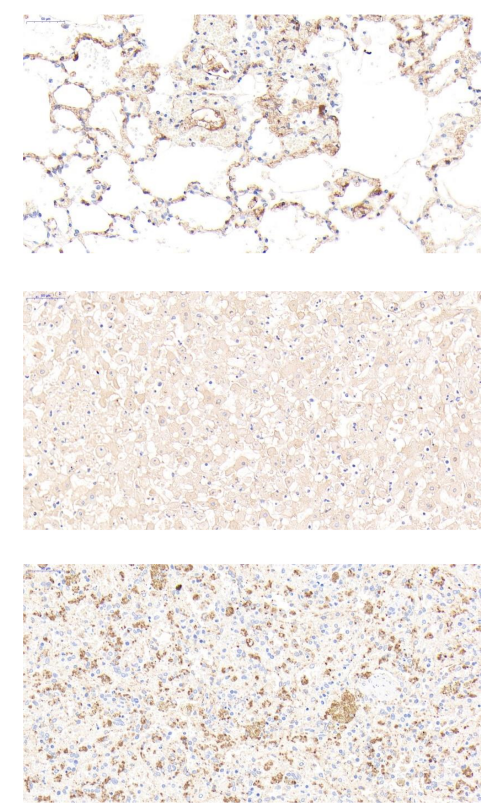

Infection 